\title{
ANÁLISE DAS SEQUENCIAS TÁTICAS OFENSIVAS DAS CATEGORIAS SUB-15 E SUB-17 DE UM CLUBE PROFISSIONAL DE FUTEBOL BRASILEIRO
}

\author{
Luan Antonio Corá Silveira \\ Universidade Estadual de Maringá, Maringá, Paraná, Brasil \\ Vanessa Menezes Menegassi \\ Universidade Estadual de Maringá, Maringá, Paraná, Brasil \\ Leandro Rechenchosky \\ Universidade Estadual de Maringá, Maringá, Paraná, Brasil \\ Augusto Oliveira \\ Coritiba Football Club, Curutiba, Paraná, Brasil \\ Wilson Rinaldi \\ Universidade Estadual de Maringá, Maringá, Paraná, Brasil \\ Paulo Henrique Borges \\ Universidade Estadual de Maringá, Maringá, Paraná, Brasil
}

\begin{abstract}
Resumo
O estudo investigou as sequências táticas ofensivas das categorias sub-15 e sub-17 de um clube de futebol profissional. A análise de 147 sequências foi feita por meio do software Match Vision Studio Premium ${ }^{\circledR}$, utilizando as variáveis propostas por Garganta (1997) e Almeida (2010). Não foram identificadas diferenças significativas nos comportamentos táticos das duas categorias. Foram verificadas correlações significativas entre o tempo de realização do ataque e o número de passes, número de jogadores, número de contatos com a bola e número de trocas de corredores $(r>0,81)$. Os resultados sugerem que há coerência do clube quanto às ideias de jogo trabalhadas com as categorias e que o tempo de realização do ataque está relacionado com o modelo de jogo destas.
\end{abstract}

Palavras-chave: Futebol. Adolescente. Esportes juvenis.

\section{OFFENSIVE TACTICAL SEQUENCES ANALYSIS OF U-15 AND U-17 CATEGO- RIES OF A PROFESSIONAL BRAZILIAN FOOTBALL CLUB}

\begin{abstract}
The study investigated the offensive tactical sequences of the under-15 and under-17 categories of a professional football club. The analysis of 147 sequences was done through the software Match Vision Studio Premium ${ }^{\circledR}$, using the variables proposed by Garganta (1997) and Almeida (2010). No significant differences were identified in tactical behaviors of the two categories. Significant correlations were verified between the time duration of attack and the number of passes, number of players, number of contacts with the ball and number of corridor changes $(r>0.81)$. The results suggest that there is coherence of the club regarding the game
\end{abstract}


ideas worked with the categories and that time duration of attack is related to their game model.

Keywords: Football. Adolescent. Youth sports.

\title{
ANÁLISIS DE LAS SECUENCIAS TÁCTICAS OFENSIVAS DE CATEGORIAS SUB- 15 Y SUB-17 DE UN CLUB PROFESIONAL DE FÚTBOL BRASILEÑO
}

\begin{abstract}
Resumen
El estudio investigó las secuencias tácticas ofensivas de las categorías sub-15 y sub-17 de un club de fútbol profesional. El análisis de 147 secuencias fue realizado por medio del software Match Vision Studio Premium ${ }^{\circledR}$, utilizando las variables propuestas por Garganta (1997) y Almeida (2010). No se identificaron diferencias significativas en los comportamientos tácticos de las dos categorías. Se verificaron correlaciones significativas entre el tiempo de realización del ataque y el número de pases, número de jugadores, número de contactos con la bola y número de cambios de corredores $(r>0,81)$. Los resultados sugieren que hay coherencia del club en cuanto a las ideas de juego trabajadas con las categorías y qué tiempo de realización del ataque está relacionado con el modelo de juego de estas.
\end{abstract}

Palabras clave: Fútbol. Adolescente. Deportes juveniles.

\section{Introdução}

Durante o jogo de futebol, as interações realizadas pelos jogadores dão corpo a uma série de comportamentos táticos que são fundamentais para buscar a excelência esportiva. A dimensão tática permite ao jogador gerir os espaços de jogo diante dos problemas apresentados dentro da partida, bem como desestruturar a dinâmica coletiva adversária (MORAES; CARDOSO; TEOLDO, 2014; TEOLDO; GUILHERME; GARGANTA, 2015).

Dentro do processo de treino-jogo, o desenvolvimento da dimensão tática não deve ser entendido como algo natural, mas sim um fenômeno construído pelas equipes a partir dos princípios táticos especiais preconizados pelo treinador e pela interação dialética deles com os jogadores, o contexto cultural e social da região ou do clube, os métodos de treinamento, entre outros (CASTELO, 1994; MACIEL, 2011). Partindo desse ponto de vista, torna-se pertinente identificar e avaliar o modelo de jogo da equipe, que é a manifestação da dimensão tática do jogo e que ocorre por meio dos comportamentos em campo dentro dos momentos de organização ofensiva, defensiva e respectivas transições. Assim, preconiza-se o desenvolvimento de um sentido coletivo em face de um conjunto de regularidades que se pretende observar, cujo horizonte seja desenvolver determinados padrões vinculados a uma morfologia comportamental (CASARIN et al., 2011).

Ao pensar o ensino de ideias relacionadas ao modelo de jogo para jovens futebolistas, espera-se que, dentro de um clube, aquilo que foi ensinado para a categoria sub-15 seja amadurecido, incorporado e aperfeiçoado pela categoria sub-17, dando continuidade ao processo de ensino-aprendizagem da modalidade, uma vez que os conteúdos tático-técnicos trabalhados durante os treinamentos devem ter um caráter de progressão complexa (BORGES et al., 2014). Ademais, a literatura tem sido consistente ao afirmar que as equipes de sucesso gastam um maior tempo elaborando suas ações ofensivas (JONES; JAMES; MELLALIEU, 2004; LAGO; MARTÍN, 2007) e que a manutenção da posse de bola pela equipe atacante pode garantir um maior número de ações ofensivas e finalizações a gol (GÓMEZ LÓPEZ; ÁLVARO, 2002).

Apesar de serem relativamente abundantes, a maioria das investigações publicadas com as temáticas supracitadas foi realizada com jogadores adultos de alto rendimento. Não 
está claro na literatura se, nas categorias de base dos clubes brasileiros, existe uma continuidade de conteúdos e semelhanças comportamentais vinculadas ao modelo de jogo ou, ainda, se o tempo gasto nas ações ofensivas relaciona-se com os indicadores tático-técnicos fundamentais para a eficácia coletiva. Essas informações são essenciais para o processo de formação esportiva, pois o desenvolvimento das dimensões físicas, técnicas, táticas e psicológicas e o seu constante monitoramento deve ser levado em consideração em um processo de formação desportiva de qualidade. Assim, os propósitos deste estudo foram: i) comparar os comportamentos táticos ofensivos dos jogadores de futebol das categorias sub-15 e sub-17 de um clube de futebol profissional; ii) analisar a relação entre o tempo de realização do ataque e os indicadores táticos coletivos.

\section{Metodologia}

A amostra foi selecionada de forma intencional e constituída por 147 sequências táticas ofensivas, realizadas por jogadores com idade entre 14 e 17 anos durante jogos do Campeonato Paranaense e Campeonato Brasileiro nas categorias sub-15 e sub-17 no ano de 2016. O estudo foi aprovado pelo comitê de ética local (Proc. 1.627.516).

As avaliações foram realizadas por meio de filmagens de jogos oficiais, com uma câmera Sony ${ }^{\circledR}$, que esteve localizada em um plano superior em relação ao plano do jogo para facilitar a visualização topográfica do campo. Após as filmagens, os vídeos foram analisados utilizando o software Match Vision Studio Premium ${ }^{\circledR}$, que tem sido empregado para a realização de estudos que recorrem à metodologia observacional (ALMEIDA, 2014; CAMERINO et al., 2012; CAMERINO; CASTANER; ANGUERA, 2015; FERNANDEZ et al., 2009; JARQUE; CAMERINO, 2012; PRATAS; VOLOSSOVITCH; FERREIRA, 2012).

Figura 1. Imagem ilustrativa do software Match Vision Studio Premium ${ }^{\circledR}$

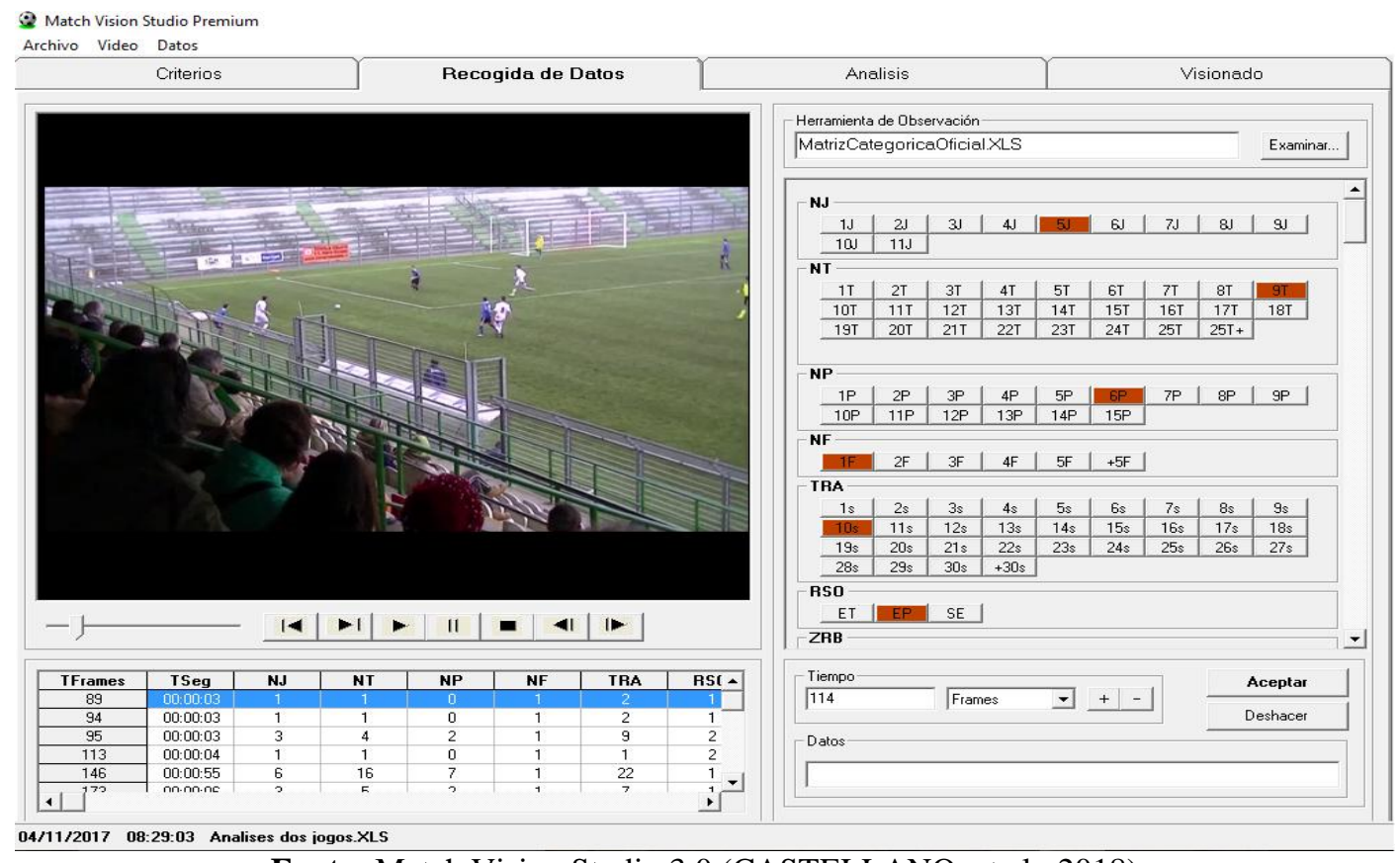

Fonte: Match Vision Studio 3.0 (CASTELLANO et al., 2018)

Para a análise dos jogos, foram recortadas as sequências táticas ofensivas das equipes que terminaram em finalização. Posteriormente, os vídeos foram inseridos no software Match Vision Studio Premium ${ }^{\circledR}$, que permite, simultaneamente à observação dos vídeos, anotar a frequência de ocorrência das variáveis selecionadas. As variáveis dependentes adotadas neste 
estudo foram sugeridas na tese doutoral de Garganta (1997) e na dissertação de Almeida (2010). Contudo, graças à flexibilidade proveniente da metodologia observacional de jogos (GRÉHAIGNE; MAHUT; FERNANDEZ, 2001) e devido à necessidade de ajustar os referidos protocolos às características dos sujeitos avaliados (adolescentes), a fim de privilegiar a validade ecológica e realçar as ações tais como são produzidas no contexto real de jogo, optou-se pelos indicadores tático-técnicos apresentados no Quadro 1.

Quadro 1: Variáveis e descrições das sequências táticas ofensivas

\begin{tabular}{|l|l|}
\hline VARIÁVEIS & DESCRIÇÕES \\
\hline $\begin{array}{l}\text { Número de jogadores } \\
\text { intervenientes (NJ) }\end{array}$ & Número de jogadores intervenientes sobre a bola durante a ação ofensiva. \\
\hline $\begin{array}{l}\text { Número de toques sobre } \\
\text { a bola (NT) }\end{array}$ & $\begin{array}{l}\text { Número total de contatos com a bola que os jogadores da equipe na fase ofensiva } \\
\text { realizam durante a jogada. }\end{array}$ \\
\hline $\begin{array}{l}\text { Número de passes reali- } \\
\text { zados (NP) }\end{array}$ & $\begin{array}{l}\text { Número total de passes efetuados com qualquer parte do corpo, que tenham sido } \\
\text { recebidos pelo companheiro de ataque, dando continuidade à fase ofensiva da } \\
\text { equipe. }\end{array}$ \\
\hline $\begin{array}{l}\text { Tempo de realização da } \\
\text { ação (TRA) }\end{array}$ & $\begin{array}{l}\text { É o tempo de duração da fase ofensiva, desde a roubada de bola/interceptação da } \\
\text { bola até a finalização da jogada. }\end{array}$ \\
\hline $\begin{array}{l}\text { Número de trocas de } \\
\text { corredores (NTC) }\end{array}$ & $\begin{array}{l}\text { Número total de vezes em que a bola trocou de corredores, segundo o campo- } \\
\text { grama de divisão do terreno de jogo em três corredores, durante a fase ofensiva. }\end{array}$ \\
\hline
\end{tabular}

Fonte: Elaborado pelos autores (2018) com variáveis de Garganta (1997) e Almeida (2010).

Após a análise, os resultados foram transportados para o software Microsoft Excel ${ }^{\circledR}$ com todos os dados coletados e tabulados.

Relativamente à análise estatística, a normalidade dos dados foi verificada por meio do teste de Kolmogorov-Smirnov. Identificada à necessidade de utilização de estatística não paramétrica, recorreu-se ao teste U-Mann Whitney para comparar os comportamentos táticos ofensivos entre as categorias sub-15 e sub-17. Posteriormente, para relacionar o tempo de realização do ataque e as variáveis ofensivas, foi utilizado o coeficiente de correlação de Spearman.

Com a finalidade de avaliar a confiabilidade das avaliações, após 21 dias das análises dos vídeos, 28,57\% (42 ações) do total das sequências ofensivas foi avaliado por um pesquisador independente e reavaliado pelo avaliador principal. Tal percentual de ações é superior ao mínimo recomendado pela literatura para esse propósito (TABACHNICK; FIDELL, 2007). Na sequência, empregou-se o coeficiente de correlação intraclasse (CCI) para variáveis quantitativas e o Índice Kappa para variáveis qualitativas. Os valores encontrados ( $\mathrm{r}>0,90 ; \mathrm{k}$ $>0,81)$ revelam boa concordância intra e interavaliadores. O nível de significância adotado foi de $5 \%$.

\section{Resultados}

A Tabela 1 apresenta os resultados da comparação entre o desempenho tático das sequências ofensivas realizadas pelas equipes sub-15 e sub-17. 
Tabela 1: Comparação do desempenho tático ofensivo entre as sequências ofensivas das categorias sub-15 e sub-17 de uma equipe brasileira

\begin{tabular}{lccc}
\hline & Sub-15 $(\mathbf{n}=\mathbf{6 3})$ & Sub-17 $(\mathbf{n}=\mathbf{8 4})$ & $\boldsymbol{p}$ \\
\cline { 2 - 3 } & $\mathbf{M d}(\mathbf{Q 1}-\mathbf{Q 3})$ & $\mathbf{M d}(\mathbf{Q 1}-\mathbf{Q 3})$ & 0,43 \\
NJ & $3,0(2,0-5,0)$ & $3,0(2,0-6,0)$ & 0,49 \\
NT & $7,0(3,0-14,0)$ & $7,5(3,0-15,0)$ & 0,77 \\
NP & $3,0(1,0-5,0)$ & $2,5(1,0-5,0)$ & 0,50 \\
TRA & $10,0(5,0-17,0)$ & $11,0(5,0-21,0)$ & 0,74 \\
NTC & $2,0(1,0-3,0)$ & $1,0(1,0-3,0)$ &
\end{tabular}

Nota: NJ = Número de jogadores que contatam a bola; NT: Número de toques realizados na bola; NP: Número de passes realizados; NF: Número de finalizações; TRA: Tempo de realização da ação; NTC: Número de trocas de corredores.

De acordo com a estatística inferencial empregada, não foram identificadas diferenças significativas nos comportamentos táticos analisados $(p>0,05)$, o que sugere uma similaridade morfológica de jogo em ambas as categorias.

A Figura 2 apresenta a correlação entre o tempo de realização da ação (TRA) e as variáveis táticas relacionadas às sequências ofensivas das equipes avaliadas.

Figura 2: Correlação entre o TRA e variáveis táticas relacionadas às sequências ofensivas
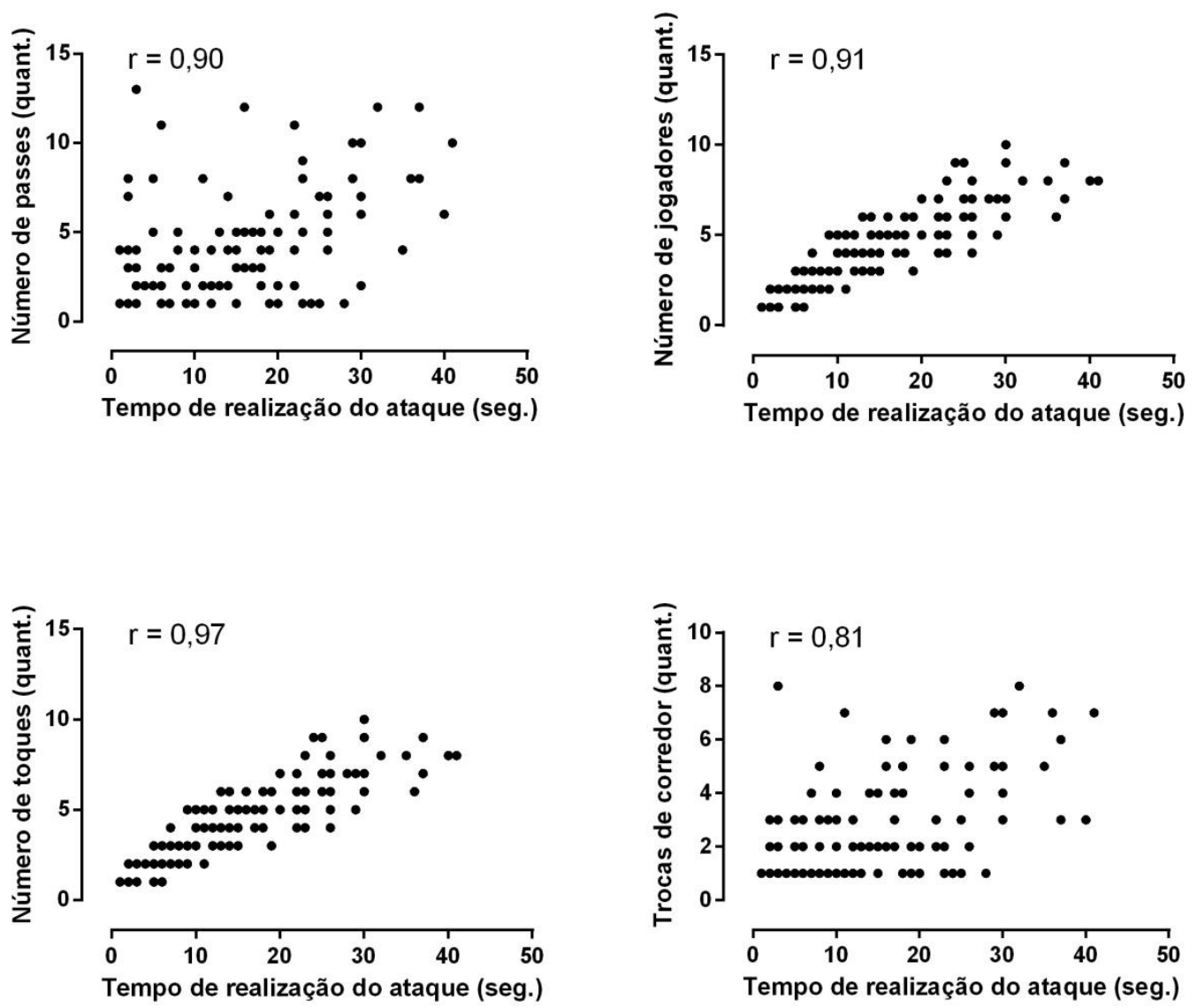

Fonte: Elaborado pelos autores, 2018. 
Conforme ilustrado na Figura 2, foram identificadas correlações significativas entre o tempo de realização do ataque e o número de passes $(p<0,01)$, o número de jogadores $(p<$ $0,01)$, o número de contatos com a bola $(p<0,01)$ e o número de trocas de corredores $(p<$ $0,02)$.

\section{Discussão}

O objetivo deste estudo foi comparar os comportamentos táticos ofensivos dos jogadores de futebol das categorias sub-15 e sub-17 de um clube de futebol profissional, assim como analisar a relação entre o tempo de realização do ataque com os indicadores táticos. Os principais resultados evidenciaram que as duas categorias apresentaram semelhanças nas sequências táticas ofensivas. Possivelmente, as ideias de jogo apresentadas pelos treinadores foram similares, o que contribui para uma continuidade dos conteúdos trabalhados da categoria sub-15 para a categoria sub-17 durante a progressão esportiva.

Essa semelhança comportamental pode contribuir com a formação de futuros talentos para o clube investigado, uma vez que, ao entender o futebol como um sistema dinâmico, devem-se mapear os comportamentos táticos (modelo de jogo) e dividi-los didaticamente ao longo do processo de formação esportiva para melhor compreensão das ideias propostas (CASARIN et al., 2011). Assim, ao transitar pelas diferentes categorias do clube, os jogadores em formação vão entender e interpretar o jogo sob uma mesma perspectiva, ainda que os pormenores relacionados ao sub-sub-princípios possam variar.

Apesar de os resultados do presente estudo apontarem para equipes que, possivelmente, interpretam a organização ofensiva através de ataques rápidos e posicionais, o estudo de Gimenes et al. (2013), que procuraram analisar as ações de contra-ataque em partidas da Copa do Mundo FIFA 2006, constatou que a maior parte dos contra-ataques envolveram três trocas de passes $(24,67 \%)$, utilizando uma variação de corredor (46,75\%), o que se assemelha com as variáveis das categorias na Tabela 1 do presente estudo. Além disso, o número de jogadores que cercam a bola influencia a decisão do tipo de passe utilizado (curto ou longo) (MALTA; TRAVASSOS, 2014), podendo colaborar com uma alteração instantânea da forma habitual de organizar uma ação ofensiva.

Já a Figura 2 apresenta correlações significativas entre o tempo de realização do ataque e o número de passes, o número de toques, o número de trocas de corredor e o número de jogadores envolvidos na jogada. Dessa forma, conforme o tempo de realização das jogadas aumentava, um acréscimo era gerado na participação dos jogadores na construção das ações, no número dos contatos entre esses jogadores sobre a bola, no número de passes executados por esses atletas nas ações ofensivas e no número de trocas de corredores durante as ações.

Embora o estudo conduzido por Tenga e colaboradores (2010) tenha evidenciado que os ataques rápidos foram mais eficazes que os ataques elaborados, acredita-se que o tempo de realização do ataque está interligado com o sucesso da equipe, uma vez que manter a posse de bola no maior tempo possível pode contribuir para a elaboração de uma jogada decisiva (GARGANTA, 1997; ANDRADE; PADILHA; TEOLDO, 2012) envolvendo mais jogadores. Não obstante, Barreira e colaboradores (2015) identificaram que os padrões de jogo vêm sofrendo influências do status da partida (vitória, empate, derrota), do estágio da competição a ser disputada, assim como do período do jogo. Portanto, outras variáveis podem influenciar decisivamente a posse de bola de uma equipe.

No estudo de Hughes e Franks (2005) sobre as Copas do Mundo de 1990 e 1994, foi verificado que as equipes que detinham um tempo maior de posse de bola culminavam em maior realização de finalizações, ou seja, obtiveram maiores oportunidades de atingir a meta. Esses achados corroboram outros dois estudos sobre a liga espanhola, em que o maior tempo 
de posse de bola foi fator determinante para as equipes melhores colocadas na primeira divisão da competição (BALLESTEROS; PEÑAS, 2010; PEÑAS; DELLAL, 2010).

Em relação à quantidade de trocas de corredor, houve similaridade na frequência de execução entre as categorias sub-15 e sub-17 (Tabela 1). A esse respeito, acredita-se que uma equipe tem mais probabilidade de ser eficiente, do ponto de vista ofensivo, quando circula a bola variando os corredores de jogo, uma vez que essa ação impõe aos jogadores de defesa a necessidade de flutuar a marcação para as diferentes faixas do gramado, e qualquer descuido ou falta de coordenação coletiva pode acarretar em espaços intersetoriais fundamentais para a exploração ofensiva (GARGANTA, 1997). Assim, Casarin et al. (2011) sinalizam a importância de incentivar, desde categorias menores, a variação de corredor do campo durante a organização ofensiva.

Os resultados encontrados enriquecem os estudos sobre a tática na formulação do modelo de jogo em equipes de jovens jogadores, independente da atribuição ou não de outros indicadores táticos ofensivos elencados com a resolução das ações. Sugere-se em novos estudos a realização de análises das sequências táticas ofensivas com mais de duas categorias de um mesmo clube ou, ainda, de outros clubes, comparando modelos de jogo de equipes da mesma cidade, estado ou nação, além de procurar comparar modelos de países distintos, atribuindo-se possíveis influências da cultura local e da região pesquisada.

\section{Conclusão}

As evidências apresentadas no trabalho sugerem que as categorias avaliadas são similares sob o ponto de vista ofensivo, o que demonstra coerência do clube quanto ao modelo de jogo adotado, bem como os conteúdos trabalhados ao longo do processo de formação esportiva. O tempo de realização do ataque apresenta-se como fator determinante para o aumento na participação dos jogadores, na frequência de passes realizados e na transição da bola entre os corredores. Essas características moldam as ações ofensivas das equipes avaliadas e refletem o modelo de jogo proposto por estas.

Como aplicação prática dos resultados encontrados, sugere-se a adoção de metodologias de ensino-aprendizagem-treinamento que estimulem a tomada de decisão e o desenvolvimento tático-técnico por meio de uma matriz conceitual, ou seja, ensinar o jogo através de ideias. Além disso, recomenda-se o diálogo dos conteúdos abordados nas diferentes faixas etárias dentro de um clube formador, para que sejam aperfeiçoados nas categorias subsequentes, conferindo, assim, um caráter de progressão complexa.

\section{Referências}

ALMEIDA, C. Caracterização das sequências ofensivas no futebol juvenil: efeitos da experiência e de variantes reduzidas do jogo. 2010. 89 f. Dissertação (Mestrado em Treino do Jovem Atleta) - Faculdade de Motricidade Humana, Universidade Técnica de Lisboa, Lisboa, 2010 .

ALMEIDA, R. Da conceptualização dos métodos de treino à operacionalização prática no quadro do modelo de jogo adotado. 2014. 99 p. Dissertação (Mestrado em Treino Desportivo) - Faculdade de Educação Física e Desporto, Universidade Lusófona de Humanidades e Tecnologias, Lisboa, 2014.

ANDRADE, M. O. C.; PADILHA, M.; TEOLDO, I. Análise da posse da bola da seleção espanhola na Copa do Mundo de Futebol FIFA - África do Sul/2010: estudo comparativo entre 
as fases classificatória e eliminatória. Revista Mineira de Educação Física, Viçosa, v. especial, n. 1, p. 2071-2079, 2012.

BALLESTEROS, J.; PEÑAS, C. Performance in team sports: identifying the keys to success in soccer. Journal of Human Kinetics, Katowice, v. 25, p. 85-91, 2010.

BARREIRA, D. et al. How elite-level soccer dynamics has evolved over the last three decades? Input from generalizability theory. Cuadernos de Psicología del Deporte, v. 15, n. 1, p. 51-62, 2015.

BORGES, P. et al. Contribuições da periodização tática para o desenvolvimento de uma identidade coletiva em equipes de futebol. Cinergis, Santa Cruz do Sul, v. 15, n. 2, p. 81-86, 2014.

CAMERINO, O.; CASTANER, M.; ANGUERA, M. Mixed methods research in the movement sciences: case studies in sport, physical education and dance. Sport, Education and Society, Cambridge, v. 20, n. 2, p. 277-282, 2015.

CAMERINO, O. et al. Dynamics of the game in soccer: detection of T-patterns. European Journal of Sport Science, London, v. 12, n. 3, p. 216-224, 2012.

CASARIN, R. et al. Modelo de jogo e processo de ensino no futebol: princípios globais e específicos. Movimento, Porto Alegre, v. 17, n. 3, p. 133-152, 2011.

CASTELLANO, J. et al. The measuring and observation tool in sports. Behavior Research Methods, v. 40, n. 3, p. 898-905, 2008.

CASTELO, J. Futebol: organização e dinâmica do jogo. Lisboa: Edições FMH, 2004.

FERNANDEZ, J. et al. Identifying and analyzing the construction and effectiveness of offensive plays in basketball by using systematic observation. Behavior Research Methods, v. 41, n. 3, p. 719-730, 2009.

GARGANTA, J. Modelação táctica do jogo de futebol: estudo da organização da fase ofensiva em equipas de alto rendimento. 1997. 318 f. Dissertação (Doutorado em Ciência do Desporto) - Faculdade de Ciências do Desporto e de Educação Física, Universidade do Porto, Porto, 1997.

GIMENES, S. et al. Modelação das ações de contra-ataque em partidas da FIFA WORD CUP 2006. Revista Brasileira de Futsal e Futebol, São Paulo, v. 5, n. 15, p. 3-14, 2013.

GÓMES LÓPEZ, M.; ÁLVARO, J. El tiempo de posesión como variable no determinante del resultado en los partidos de fútbol. El Entrenador Español, v. 97, n. 1, p. 39-47, 2002.

GRÉHAIGNE, J.F.; MAHUT, A.; FERNANDEZ, A. Qualitative observational tools to analyse soccer. International Journal of Performance Analysis in Sport, v. 1, n. 1, p. 52$61,2001$.

HUGHES, M.; FRANKS, I. Analysis of passing sequences, shots and goals in soccer. Journal of Sports Sciences, v. 23, n. 5, p. 509-514, 2005. 
JARQUE, D.; CAMERINO, O. Eficacia de los sistemas ofensivos enbalon mano. Apunts. Educación Física y Deportes, Barcelona, v. 2, n. 108, p. 70-81, 2012.

JONES, P.D.; JAMES, N.; MELLALIEU, S. D. Possession as a performance indicator in soccer. International Journal of Performance Analysis in Sport, v. 4, n. 1, p. 98-102, 2004.

LAGO, C.; MARTÍN, R. Determinants of possession of the ball in soccer. Journal of Sports Science, v. 25, n. 9, p. 969-974, 2007.

MACIEL, J. Não o deixes matar: o bom futebol e quem o joga. Lisboa: Chiado Editora, 2011.

MALTA, P.; TRAVASSOS, B. Caracterização da transição defesa-ataque de uma equipa de futebol. Motricidade, Vila Real, v. 10, n. 1, p. 27-37, 2014.

MORAES, E. L.; CARDOSO, F.; TEOLDO, I. Análise dos padrões ofensivos da Seleção Espanhola de Futebol na Copa do Mundo FIFA® 2010 em relação ao "status" da partida. Revista Brasileira de Educação Física e Esporte, São Paulo, v.28, n.3, p.361-369, 2014.

PEÑAS, C.; DELLAL, A. Ball possession strategies in elite soccer according to the evolution of the match-score: the influence of situational variables. Journal of Human Kinetics, Katowice, v. 25, p. 93-100, 2010.

PRATAS, J.; VOLOSSOVITCH, A.; FERREIRA, P. The effect of situationalvariables on teams' performance in offensive sequences ending in a shot on goal, a case study. The Open Sports Sciences Journal, Sharjah, v. 5, p. 193-199, 2012.

TABACHNICK, B.; FIDELL, L. Using multivariate statistics. Londres: Pearson Education, 2007.

TENGA, A. et al. Effect of playing tactics on goal scoring in Norwegian professional soccer. Journal of Sports Sciences, v. 28, n. 3, p. 237-244, 2010.

TEOLDO, I.; GUILHERME, J.; GARGANTA, J. Para um futebol jogado com ideias: concepção, treinamento e avaliação do desempenho tático de jogadores e equipes. Vila Mariana: Appris editora, 2015. $319 \mathrm{p}$.

Recebido em: 06/01/2018

Revisado em: 03/05/2018

Aprovado em: 05/05/2018

Endereço para correspondência:

luan-antonio.silveira@hotmail.com

Luan Antonio Corá Silveira

Universidade Estadual de Maringá, Centro de Ciências da Saúde.

Avenida Colombo, Zona 7

87020-000 - Maringa, PR - Brasil 